\title{
Ecocardiografia modo Doppler pulsado em gatos clinicamente sadios
}

\author{
[Pulsed wave Doppler echocardiography in clinically healthy cats] \\ R.O. Carvalho, R.B. Araújo, E.F. Silva \\ Escola de Veterinária - UFMG \\ Caixa Postal 567 \\ 30123-970 - Belo Horizonte, MG
}

\begin{abstract}
RESUMO
Estudou-se o fluxo sangüíneo através das quatro valvas cardíacas em 30 gatos clinicamente sadios, com idade entre um e cinco anos e peso médio de $4,08 \mathrm{~kg}$, por meio da ecocardiografia modo Doppler pulsado. Foram medidas a velocidade máxima e a velocidade média dos fluxos, e realizou-se uma análise qualitativa dos seus perfis. Os animais foram sedados pela combinação de quetamina $(12 \mathrm{mg} / \mathrm{kg}) \mathrm{e}$ acepromazina $(0,04 \mathrm{mg} / \mathrm{kg})$, aplicados por via intramuscular. Observou-se correlação positiva entre os parâmetros avaliados e a freqüência cardíaca, com exceção daqueles medidos no fluxo da valva aórtica. Não se observou correlação entre velocidades máxima e média e freqüência cardíaca e entre aquelas e peso corporal, e não houve diferença entre sexos.
\end{abstract}

Palavras-chave: gato, Doppler pulsado, ecocardiografia, fluxo sangüíneo

\begin{abstract}
Pulsed wave Doppler echocardiography was used to study blood flow across the cardiac valves in 30 five-year-old cats (average body weight $=4.08 \mathrm{~kg}$ ). Animals were sedated using a combination of ketamin $(12 \mathrm{mg} / \mathrm{kg}, \mathrm{IM})$ and acepromazin $(0.04 \mathrm{mg} / \mathrm{kg}$, IM). Peak and mean velocities were determined, and blood flow patterns were recorded at the four cardiac valves. All variables, except those characterizing aortic valve flow, were positively correlated with heart rate. Blood flow variables were not correlated, however, with body weight; and there were no differences between males and females.
\end{abstract}

Keywords: cat, pulsed Doppler, echocardiography, cardiac blood flow

\section{INTRODUÇÃO}

A ecocardiografia representa um grande avanço na cardiologia médica e veterinária, possibilitando a avaliação morfofuncional do coração de forma não-invasiva. Por tratar-se de um método inócuo e minimamente estressante, é ideal em situações de risco e para acompanhamento da progressão das doenças e da resposta aos tratamentos (Yuill e O'Grady, 1991; Kittleson e Kienle, 1998). O exame é constituído por três modalidades: modo-M, bidimensional e Doppler. Com os dois primeiros, podem-se

Recebido em 26 de julho de 2004

Aceito em 19 de outubro de 2005

*Autor para correspondência (corresponding author)

E-mail: baracat@vet.ufmg.br visibilizar os átrios, ventrículos, aurículas, valvas cardíacas e os grandes vasos, obtendo-se imagens dinâmicas que permitem avaliar a espessura das paredes em sístole e diástole, o movimento muscular, valvular e os índices de contratilidade do coração. O modo Doppler possibilita o estudo da direção e velocidade do fluxo em pontos-chave anatômicos, além de detectar a presença de fluxos turbulentos no coração e nos grandes vasos. Essas informações, interpretadas em conjunção com outros achados ecocardiográficos, podem ser usadas para identificar padrões anormais de fluxo (shunting, refluxos e estenoses valvares), para acessar a função sistólica e diastólica do coração e quantificar a gravidade das lesões (Bonagura et al., 1998; Muzzi, 2002). 
$\mathrm{Na}$ clínica de felinos, esse exame é um importante método auxiliar que contribui para o diagnóstico de doenças cardíacas, muitas vezes prejudicado pela alta freqüência cardíaca, pelo ronronar e pelas variações anatômicas de posicionamento cardíaco inerentes à espécie (Pipers e Hamlin, 1980). Dentre as alterações cardíacas que acometem os felinos, a miocardiopatia hipertrófica $(\mathrm{MCH})$ é considerada uma das mais importantes (Atkins et al., 1992). As alterações hemodinâmicas responsáveis pelos sinais clínicos da $\mathrm{MCH}$ são detectadas pelo exame Doppler dos fluxos intracardíacos (Bright et al., 1999).

Fluxos anormais de alta velocidade são encontrados em muitas alterações cardíacas, incluindo estenose das valvas, obstrução dinâmica da via de saída do ventrículo, defeito de septo interventricular, insuficiências valvulares e persistência de ducto arterioso (Moise, 1989; Yuill e O’ Grady, 1991; Bonagura et al., 1998). Para determinar a gravidade desses aumentos de velocidade, é essencial o conhecimento das velocidades máximas através das valvas em corações normais. O objetivo deste estudo foi determinar as velocidades máximas e médias dos fluxos intracardíacos e descrever seus perfis em gatos clinicamente normais.

\section{MATERIAL E MÉTODOS}

Foram utilizados 30 gatos (15 machos e 15 fêmeas) clinicamente sadios, sendo 28 sem padrão racial definido e dois da raça Persa, com idade compreendida entre um e cinco anos, e peso médio de $4,08 \pm 1,02 \mathrm{~kg}$. Os animais foram selecionados de acordo com os critérios: ausência de história de doença clínica passada ou atual, auscultação dentro da normalidade, avaliação pressórica e eletrocardiográfica dentro dos padrões normais, ausência de indícios clínicos e radiográficos de doença cardiopulmonar (Fox et al., 1985; Santilli e Bussadori, 1998).

Os animais foram submetidos a exame clínico geral e específico e, em seguida, foram feitas avaliações eletrocardiográfica ${ }^{1}$, radiográfica ${ }^{2}$ e pressórica por método Doppler vascular ${ }^{3}$. O

\footnotetext{
- Eletrocardiógrafo Ecafix ECG40 - Funbec

- Aparelho de raio-x VMI 500ma

- Parks Medical - Model 811-B
}

objetivo desses exames foi excluir animais que porventura apresentassem doenças cardíacas que alterassem os resultados da pesquisa. A freqüência respiratória (FR) foi medida com os animais em repouso e a freqüência cardíaca (FC), durante a eletrocardiografia (ECG). Essas medidas foram realizadas novamente durante $o$ exame ecocardiográfico.

Os gatos foram depilados na região ventral do tórax e em pequenas áreas na extremidade distal dos membros anteriores e posterior esquerdo, onde foram colocados eletrodos adesivos ${ }^{4}$ para acompanhamento eletrocardiográfico simultâneo. Em seguida, foram sedados com cloridrato de quetamina $(12 \mathrm{mg} / \mathrm{kg})$ e maleato de acepromazina $(0,04 \mathrm{mg} / \mathrm{kg})$ por via intramuscular (DeMadron et al., 1985; Moise et al., 1986), os olhos foram vendados com máscara, e os ouvidos tampados com algodão a fim de isolá-los do ambiente. Passados 10 minutos da aplicação, tempo esperado para estabilização das freqüências cardíaca e respiratória, foram, então, posicionados inicialmente em decúbito lateral esquerdo e depois direito, sobre uma mesa apropriada com abertura retangular, de forma que o transdutor ficasse posicionado sob a área cardíaca. Foi utilizado um transdutor de $5 \mathrm{mHz}$ do aparelho de ecodopplercardiografia ${ }^{5}$, coberto com uma densa camada de gel acústico ${ }^{6}$. Os exames foram gravados em videoteipe ${ }^{7}$ e impressos $^{8}$ em papel termossensível. ${ }^{9}$ Foram realizadas cinco medidas em diferentes ciclos cardíacos para estabelecer a média de cada parâmetro para cada animal. Todos os animais foram avaliados pelo mesmo examinador.

Utilizou-se o Doppler pulsado para obtenção dos fluxos através das valvas mitral, tricúspide, aórtica e pulmonar, e, para o posicionamento do transdutor, seguiram-se as recomendações de Yuill e O'Grady (1991), Kirberger et al. (1992a), Darke et al. (1993) e Boon (1998). O alinhamento do feixe de ultra-som com o fluxo foi guiado pela imagem bidimensional, pelo acompanhamento do registro e pelo sinal de áudio emitido pelo aparelho (Morcerf, 1996; Kittleson e Kienle, 1998).

\footnotetext{
4 - Eletrodos para ECG AG/AGCL com gel - Embramac

${ }^{5}$ Sonos 100CF - Hewlett Packard

${ }^{6}$ Gel para transmissão ultrassônica-In Shape

${ }^{7}$ Vídeo-gravador Sharp VC 1900

${ }^{8}$ Video graphic printer UP-890

${ }^{9}$ Printing paper Sony UPP - 110HA Type IV
} 
A valva aórtica foi avaliada na visão paraesternal esquerda, apical cinco câmaras, com o volume de amostra posicionado imediatamente após a valva. Para a valva pulmonar, utilizou-se a visão paraesternal direita transversal na altura da valva aórtica. $\mathrm{O}$ fluxo da valva tricúspide foi estudado na visão paraesternal esquerda apical quatro câmaras ou na visão intermediária entre a apical quatro câmaras e a paraesternal transversal esquerda com átrio e aurícula direitos. O fluxo da valva mitral, por sua vez, foi examinado na visão paraesternal esquerda, apical quatro câmaras ou apical cinco câmaras. Para cada valva, foram medidas a velocidade máxima da curva (Vmax) e a sua velocidade média (Vmed), efetuando-se cinco medições para cada variável e calculandose a sua média.

Os dados foram analisados pela estatística descritiva, calculando-se a média, o desviopadrão e o coeficiente de variação para cada variável. As medidas obtidas foram comparadas com o peso corporal e com a freqüência cardíaca dos animais, pela análise de correlação de Pearson. Utilizou-se o teste $\mathrm{t}$ de student para comparação de médias entre machos e fêmeas.

\section{RESULTADOS}

O peso médio dos animais foi de $4,08 \pm 1,02 \mathrm{~kg}$. Os machos apresentaram-se mais pesados $(\mathrm{t}=2,54, \quad \mathrm{P}<0,05)$, com média de $4,8 \pm 0,93 \mathrm{~kg}$ contra $3,4 \pm 0,55 \mathrm{~kg}$ das fêmeas. A freqüência cardíaca média foi de $185 \pm 26$ batimentos por minuto (bpm) durante o ECG, aumentando para $210 \pm 29 \mathrm{bpm}$ durante o exame ecocardiográfico. A freqüência respiratória média durante $o$ repouso foi de $23 \pm 3$ movimentos respiratórios por minuto (mrm), passando para $26 \pm 7 \mathrm{mrm}$ durante o exame ecocardiográfico.

O fluxo da valva aórtica foi negativo durante a sístole, apresentando-se pontiagudo, com fase de aceleração rápida e laminar e desaceleração mais lenta com maior espectro de dispersão das velocidades, formando um triângulo invertido assimétrico (Fig. 1). Não houve diferença relacionada à respiração. Não se observou correlação entre as variáveis Vmax e Vmed e a freqüência cardíaca ou peso corporal, e não houve diferença entre os sexos.

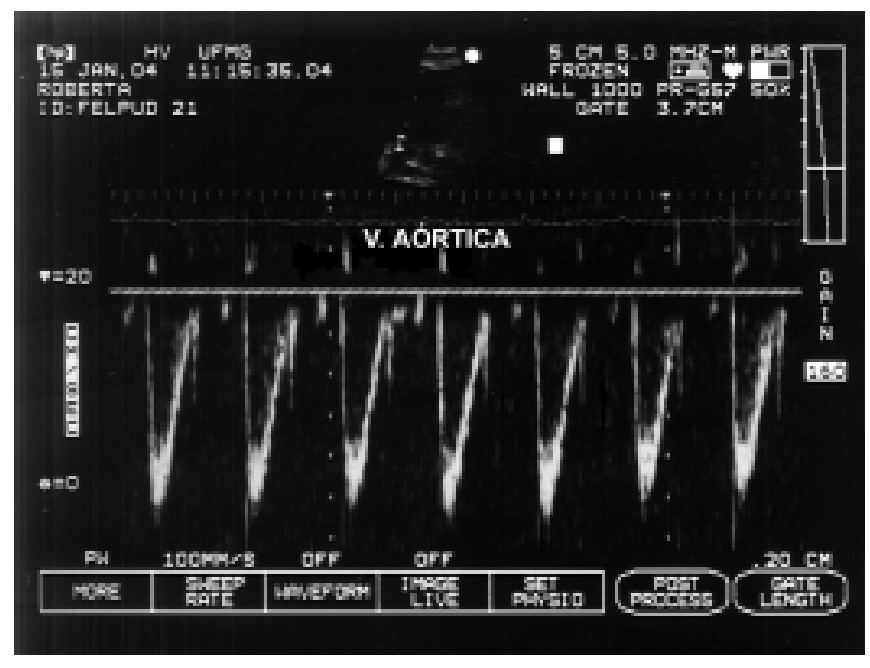

Figura 1. Fluxo da valva aórtica. Modo Doppler pulsado. O fluxo foi obtido a partir da visão paraesternal esquerda, apical cinco câmaras.

$\mathrm{Na}$ valva pulmonar, observou-se um fluxo sistólico negativo, laminar, com dispersão maior do espectro durante a velocidade máxima e a desaceleração, porém menos pontiagudo e mais simétrico em relação ao fluxo da valva aórtica
(Fig. 2). A velocidade do fluxo variou com a respiração em $21 \%$ dos gatos, aumentando discretamente durante a inspiração. Em 43\% dos animais, houve interferência do pulmão sobre a imagem bidimensional, de forma intermitente, 
coincidindo com o desaparecimento ou deformação do fluxo no monitor e alteração do sinal de áudio. Em 21\% dos animais, observouse fluxo diastólico negativo, de baixa velocidade e curta duração. Observou-se correlação entre a freqüência cardíaca e os parâmetros Vmax e Vmed, e não se observou relação linear entre as variáveis e o peso corporal ou sexo.

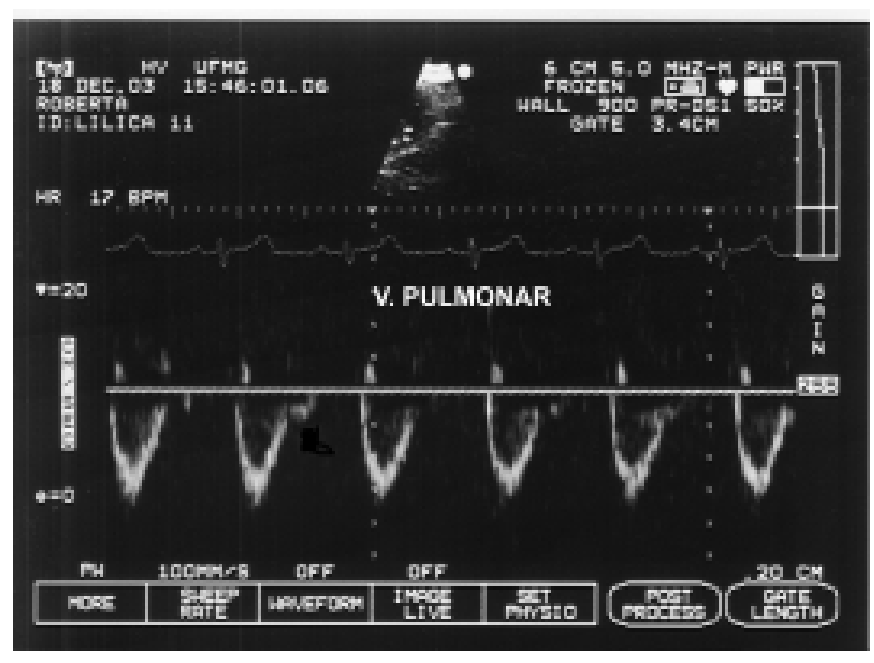

Figura 2. Fluxo da valva pulmonar. Modo Doppler pulsado. O fluxo foi obtido a partir da visão paraesternal direita transversal, vasos da base.

O fluxo através da valva mitral foi positivo durante a diástole, laminar, de formato triangular pontiagudo e monofásico, com exceção de três gatos $(10 \%)$, nos quais as ondas $\mathrm{E}$ e $\mathrm{A}$ apresentaram-se parcialmente unidas, sem diastase, com maior velocidade na onda E (Fig. 3). Em $30 \%$ dos gatos, observou-se fluxo sistólico positivo, de baixa velocidade e curta duração. Observou-se correlação entre a freqüência cardíaca e as velocidades medidas, mas não foram observadas associações com peso corporal ou sexo. Não foi observado efeito da respiração na velocidade do fluxo transmitral.

Durante a diástole, o fluxo da valva tricúspide foi positivo, com formato que variou de um triângulo pontiagudo a uma aparência semelhante a de uma chama (Fig. 4). As ondas E e A apresentaram-se completamente unidas, com exceção de oito gatos $(26,6 \%)$, em que se observou fusão quase total. Nesses animais, a velocidade máxima foi maior na onda $E$. Observou-se fluxo sistólico positivo, de curta duração e baixa velocidade em $76 \%$ dos gatos. A respiração afetou a velocidade do fluxo em $66 \%$ dos animais, com a inspiração resultando em velocidades maiores. Observou-se correlação entre as variáveis e a freqüência cardíaca e, novamente, não houve associação entre a velocidade dos fluxos e o peso corporal ou diferença entre os sexos.

Os valores da média, desvio-padrão e coeficiente de variação das características estudadas encontram-se na Tab. 1.

Tabela 1. Médias, desvios-padrão e coeficientes de variação da velocidade máxima e velocidade média dos fluxos através das valvas cardíacas em gatos clinicamente sadios

\begin{tabular}{|c|c|c|c|c|}
\hline Variável & $\overline{\mathrm{x}} \pm \mathrm{s}$ & $\begin{array}{l}\text { Coeficiente } \\
\text { de variação }\end{array}$ & $\begin{array}{c}\text { Correlação } \\
\text { com FC }\end{array}$ & $P$ \\
\hline \multicolumn{5}{|l|}{ Valva aórtica } \\
\hline $\operatorname{Vmax}(\mathrm{m} / \mathrm{s})$ & $0,85 \pm 0,13$ & 15,3 & ns & - \\
\hline Vmed $(\mathrm{m} / \mathrm{s})$ & $0,52 \pm 0,07$ & 13,5 & ns & - \\
\hline \multicolumn{5}{|c|}{ Valva pulmonar } \\
\hline $\operatorname{Vmax}(\mathrm{m} / \mathrm{s})$ & $0,82 \pm 0,16$ & 19,5 & 0,45 & $<0,05$ \\
\hline Vmed (m/s) & $0,55 \pm 0,11$ & 20,0 & 0,40 & $<0,05$ \\
\hline \multicolumn{5}{|l|}{ Valva mitral } \\
\hline $\operatorname{Vmax}(\mathrm{m} / \mathrm{s})$ & $0,83 \pm 0,16$ & 19,3 & 0,65 & $<0,01$ \\
\hline Vmed (m/s) & $0,50 \pm 0,09$ & 18 & 0,62 & $<0,01$ \\
\hline \multicolumn{5}{|c|}{ Valva tricúspide } \\
\hline $\operatorname{Vmax}(\mathrm{m} / \mathrm{s})$ & $0,64 \pm 0,14$ & 21,9 & 0,68 & $<0,01$ \\
\hline Vmed $(\mathrm{m} / \mathrm{s})$ & $0,40 \pm 0,07$ & 17,5 & 0,57 & $<0,01$ \\
\hline
\end{tabular}




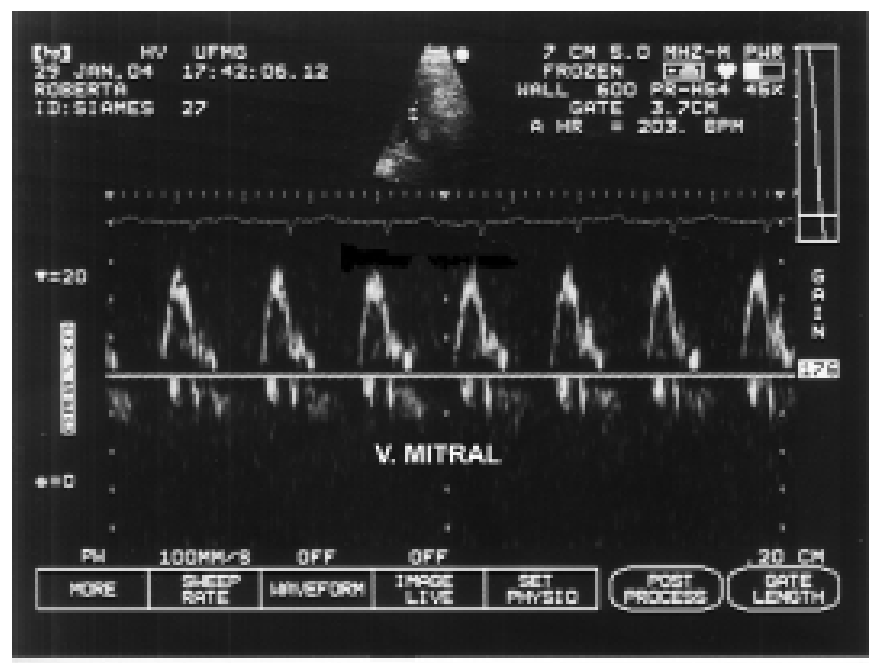

Figura 3. Fluxo da valva mitral. Modo Doppler pulsado. O fluxo foi obtido a partir da visão paraesternal esquerda, apical cinco câmaras.

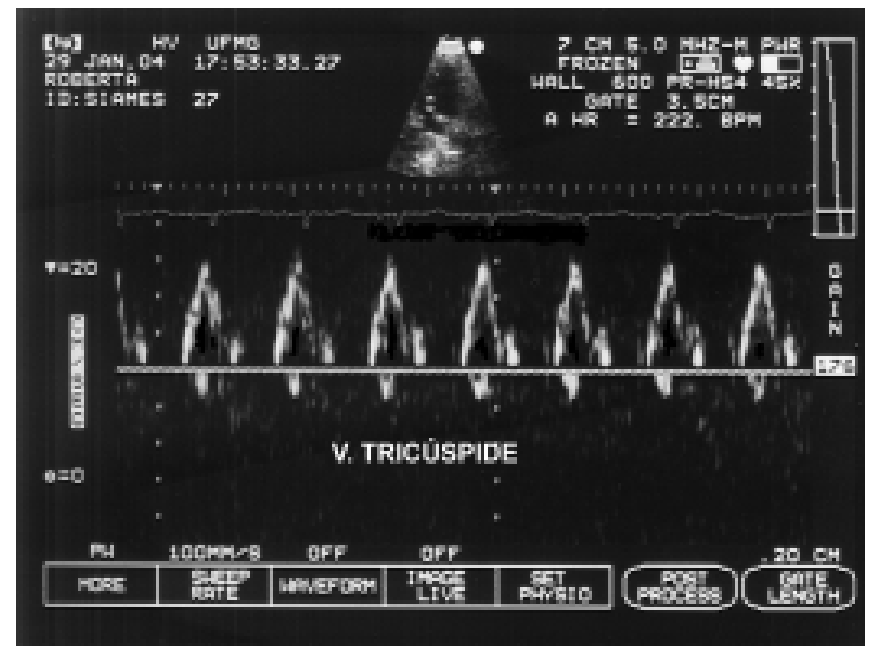

Figura 4. Fluxo da valva tricúspide. Modo Doppler pulsado. O fluxo foi obtido a partir da visão paraesternal esquerda, apical quatro câmaras.

\section{DISCUSSÃO}

Apesar da alteração provocada por tranqüilizantes na freqüência cardíaca, contratilidade e diferentes condições de enchimento ventricular, devido à extensa duração do exame e à resistência freqüente dos gatos a qualquer forma de contenção, foi necessário o uso de sedação para obter imagens tecnicamente adequadas, conforme relataram Pipers e Hamlin
(1980), DeMadron et al. (1985), Jacobs e Knight (1985) e Bonagura et al. (1998).

Observou-se aumento significativo da freqüência cardíaca após a sedação, já descrito por DeMadron et al. (1985) e Jacobs e Knight (1985). De acordo com Trim (1987), o aumento deve-se à ação simpatomimética da quetamina, que também eleva o débito cardíaco e a pressão arterial. Todavia, a freqüência média observada 
foi menor que a obtida por Skrodzki e Schille (1999) durante o exame ecocardiográfico em gatos não sedados (diferença de 25,7\%). Segundo Hamlin (1989), isso acontece porque os animais acordados são submetidos a estresse psíquico muito grande durante o exame, com aumento considerável da freqüência cardíaca.

Trim (1987) relatou que a acepromazina exerce mínimo efeito no sistema cardio-respiratório de gatos saudáveis, além de ser antiarrítmica, razão do seu uso neste estudo. Um bom efeito tranqüilizante foi obtido com a associação de quetamina e acepromazina, com rápido início de ação e duração aproximada de 40 minutos.

Os fluxos obtidos pelo modo Doppler apresentaram-se laminares e bem definidos, conforme descrito por Bonagura et al. (1998). Após o posicionamento inicial do volume de amostra guiado pelo eco bidimensional, alinhando-se ao máximo o transdutor paralelamente à direção presumível do fluxo, utilizou-se o sinal de áudio ou o próprio registro do Doppler para captar a maior velocidade do fluxo, como recomendado por Morcerf (1996) e Kittleson e Kienle (1998). Foram obtidos fluxos de boa qualidade, com exceção de 10 gatos $(33,3 \%)$, nos quais houve maior dispersão do espectro de velocidades no fluxo da valva pulmonar, sem contudo interferir nas características avaliadas. Skrodzki e Schille (1999), ao avaliarem o fluxo aórtico, encontraram problema semelhante e citaram uma discrepância entre o bom posicionamento do feixe, como mostrado na imagem bidimensional, e os sinais recebidos após a comutação para o modo Doppler. Neste estudo, como o fluxo pulmonar foi registrado ao fim do exame e após a mudança do decúbito lateral esquerdo para o direito, os animais já começavam a reagir com movimentos e aumento da freqüência cardíaca, piorando a qualidade da imagem e dificultando o alinhamento do transdutor e o posicionamento adequado do volume de amostra.

A direção, a coordenação temporal em relação ao ECG e a intensidade dos sinais nos quatro fluxos estudados foram semelhantes aos descritos em cães por Kirberger et al. (1992a), Muzzi (1998) e Boon (1998). Diferente dessa espécie, porém, o perfil do fluxo diastólico dos gatos apresentou-se monofásico nas valvas mitral e tricúspide, em $90 \%$ e $73,3 \%$ dos exames, respectivamente, semelhante ao descrito por Skrodzki e Schille (1999). Esses autores citaram a alta freqüência cardíaca dos felinos como causa desse fenômeno, que foi raras vezes observado em cães por Kirberger et al. (1992a), mas relatado por Nishimura et al. (1989), em humanos. Alves et al. (2001), ao estudarem cães neonatos, observou essa fusão das ondas $\mathrm{E}$ e $\mathrm{A}$ em freqüências superiores a $230 \mathrm{bpm}$. Domanjko e Thomas (1996), citados por Skrodzki e Schille (1999), observaram a fusão das ondas $\mathrm{E}$ e A em freqüências cardíacas maiores que 190bpm. Santilli e Bussadori (1998), ao trabalharem com gatos cooperativos e calmos, conseguiram a separação das ondas E e A em freqüências de até $170 \mathrm{bpm}$. Nesta pesquisa, essa fusão ocorreu a partir de $175 \mathrm{bpm}$ na valva tricúspide e acima de 180 na mitral. Apenas um gato, com $183 \mathrm{bpm}$, apresentou separação parcial entre as ondas E e A da valva tricúspide. Quanto menor a freqüência cardíaca, tanto mais longa foi a diástole, e o caráter bifásico do fluxo tornou-se mais visível, evidenciando o pico $\mathrm{E}$ maior que o A, conforme relatado por Skrodzki e Schille (1999). Todavia, nos animais cujo perfil foi bifásico no fluxo das valvas mitral e tricúspide, a velocidade máxima da onda $\mathrm{A}$ foi bem próxima à da onda E. Esse resultado sugere que a contração do átrio contribuiu em grande parte para o enchimento do ventrículo, como também observou Alves et al. (2001) em cães neonatos, nos quais tal fenômeno ocorre naturalmente devido à fisiologia neonatal. Quando o fluxo apresentou-se bifásico, a Vmax foi medida pela velocidade da onda $\mathrm{E}$.

Como observado por Kirberger et al. (1992a) e Muzzi (1998), registrou-se fluxo sistólico positivo nas valvas tricúspide e mitral em $76 \%$ e $30 \%$ dos gatos, respectivamente. Skrodzki e Schille (1999) descreveram-no em 8,9\% dos exames, apenas na valva mitral. Adicionalmente, durante a diástole, observou-se fluxo negativo na valva pulmonar em $21 \%$ dos animais, também registrado pelos mesmos autores, que consideraram tais fluxos como sendo normais.

Em relação à analise quantitativa do fluxo, pesquisou-se a existência de associação entre peso corporal, freqüência cardíaca e os parâmetros avaliados. Não se verificou relação linear entre a velocidade dos fluxos e o peso corporal, provavelmente devido à uniformidade de peso nos animais examinados. As variáveis 
estudadas correlacionaram-se positivamente com a freqüência cardíaca no fluxo das valvas mitral, tricúspide e pulmonar. Estes resultados são diferentes dos citados por Skrodzki e Schille (1999), que observaram correlação positiva entre a freqüência cardíaca e a Vmax e Vmed somente no fluxo transmitral. Kirberger et al. (1992b) registraram aumento na velocidade do fluxo nas quatro valvas, associado ao aumento da freqüência cardíaca, enquanto Yuill e O'Grady (1991) não verificaram nenhuma associação entre esses parâmetros. Em humanos, Harrison et al. (1991) e Yu e Sanderson (1997) verificaram que a velocidade do pico $\mathrm{A}$ da mitral é influenciada positivamente pela freqüência cardíaca, assim como Santilli e Bussadori (1998) observaram em gatos. Neste trabalho não foi possível essa comparação devido à fusão das ondas E e A na maioria dos animais.

A influência da respiração na velocidade do fluxo foi observada nas valvas pulmonar e tricúspide, como também observado em cães por Kirberger et al. (1992a) e Muzzi (1998). Skrodzki e Schille (1999) observaram essa influência em apenas quatro gatos com idade inferior a três meses, no fluxo da valva tricúspide. De acordo com Bonagura et al. (1998), essa variação pode ser explicada pelas alterações que ocorrem no enchimento ventricular durante a ventilação pulmonar ou, ainda, devido ao movimento do coração durante a respiração, que desloca o feixe ultra-sônico para a periferia da artéria pulmonar.

A velocidade máxima no fluxo das valvas mitral e tricúspide foi semelhante aos valores encontrados por Skrodzki e Schille (1999). Domanjko e Thomas (1996), citados por Skrodzki e Schille (1999), também encontraram média semelhante para a Vmax da tricúspide. Divergindo disso, esses autores encontraram médias $22,3 \%$ maiores e $12,9 \%$ menores, respectivamente, para a média da Vmax no fluxo aórtico. Para a Vmax do fluxo pulmonar, os mesmos autores obtiveram média $15,8 \%$ maior. Santilli e Bussadori (1998), Bright et al. (1999) e Domanjko e Thomas (1996), citados por Skrodzki e Schille (1999), encontraram valor menor para a Vmax da valva mitral $(15,7 \%$, $16,8 \%$ e $19,3 \%$, respectivamente). Suspeita-se que a angulação entre o feixe de ondas emitidas e a direção do fluxo tenha diferido entre os examinadores, sendo a responsável pelas diferenças encontradas nas velocidades dos fluxos, como sugerido por Skrodzki e Schille (1999). Além disso, esses últimos autores adotaram a visão paraesternal esquerda transversal ao avaliarem o fluxo da valva pulmonar, o que também pode explicar as diferenças encontradas, como informaram Yuill e O’Grady (1991). Uma outra explicação possível é o aumento do tônus simpático provocado pela quetamina, influenciando os picos de velocidade, como descrito por Bonagura et al. (1998).

Quanto à Vmed, seu valor foi semelhante ao encontrado por Skrodzki e Schille (1999) apenas no fluxo da valva pulmonar. As outras medidas apresentaram diferenças superiores a $10 \%$.

\section{CONCLUSÕES}

É possível obter fluxos valvares com perfil adequado às análises do modo Doppler em felinos sedados com quetamina e acepromazina. Os parâmetros da ecocardiografia Doppler em felinos devem ser correlacionados com a freqüência cardíaca.

\section{REFERÊNCIAS BIBLIOGRÁFICAS}

ALVES, R.O.; ARAÚJO, R.B.; SILVA, E.F. et al. Ecocardiografia Doppler em cães neomatos. Arq. Bras. Med. Vet. Zootec., v.53, p.413-419, 2001

ATKINS, C.E.; GALLO, A.M. KURZMAN, I.D. et al. Risk factors, clinical signs, and survival in cats with a clinical diagnosis of idiopathic hypertrophic cardiomyopathy: 74 cases (1985-1989). J. Am. Vet. Med. Assoc., v.201, p.613-618, 1992.

BONAGURA, J.D.; MILLER, M.W.; DARKE, P.G.G. Doppler echocardiography I: pulsed-wave and continuous-wave examinations. Vet. Clin. North. Am., v.28, p.1325-1359, 1998.

BOON, J.A. The echocardiographic examination: spectral Doppler. In: Manual of veterinary echocardiography. Baltimore: Willians \& Wilkins, 1998. cap.2, p.112-128.

BRIGHT, J.M.; HERRTAGE, M.E.; SCHNEIDER, J.F. Pulsed Doppler assessment of left venticular diastolic function in normal and cardiomyopathic cats. J. Am. Anim. Hosp. Assoc., v.35, p.285-291, 1999. 
DARKE，P.G.G.; BONAGURA，J.D.; MILLER, M. Transducer orientation for Doppler echocardiography in dogs. J. Small Anim. Pract., v.34, p.2-8, 1993.

DEMADRON, E.; BONAGURA, J.D.; HERRING, D.S. Two-dimensional echocardiography in the normal cat. Vet. Radiol., v.26, p.149-158, 1985.

DOMANJKO, A.; THOMAS, W.P. Pulsed wave Doppler echocardiography in normal cats. Proc. Europ. Soc. Vet. Cardiol. Free Comun., 1996. (Abstract).

FOX, P.R.; BOND, B.R.; PETERSON, M.E. Echocardiographic reference values in healthy cats sedated with ketamine hydrochloride. Am. J. Vet. Res., v.46, p.1479-1484, 1985.

HAMLIN, R.L. Heart rate of the cat. J. Am. Anim. Hosp. Assoc., v.25, p.284-286, 1989.

HARRISON, M.R.; CLIFTON, D.; PENNEL, A.T. et al. Effect of heart rate on left ventricular diastolic transmitral flow velocity patterns assessed by Doppler echocardiography in normal subjects. Am. J. Cardiol., v.67, p.622-627, 1991.

JACOBS, G.J.; KNIGHT, D.H. Change in M-mode echocardiographic values in cats given ketamine. Am. J. Vet. Res., v.46, p.1712-1713, 1985.

KIRBERGER, R.M.; BERG, P.B.; DARAZ, B. Doppler echocardiography in the normal dog: part I - velocity findings and flow patterns. Vet. Radiol. Ultrasound, v.33, p.370-379, 1992a.

KIRBERGER, R.M.; BERG, P.B.; DARAZ, B. Doppler echocardiography in the normal dog: part II- factors influencing blood flow velocities and a compaison between left and right heart blood flow. Vet. Radiol. Ultrasound, v.33, p.380-386, 1992b.

KITTLESON, M.D.; KIENLE, R.D. Echocardiography. In:_ Small animal cardiovascular medicine. St. Louis: Mosby, 1998. cap.6, p.95-117.

MOISE, N.S. Doppler echocardiographic evaluation of congenital cardiac disease. J. Vet. Intern. Med., v.3, p.195-207, 1989.

MOISE, N.S.; HORNE, W.A.; FLANDERS, J.A. et al. Repeatability of the M-mode echocardiogram and the effects of acute changes in heart rate, cardiac contractility, and preload in healthy cats sedated with ketamine hydrochloride and acepromazine. Cornell Vet., v.76, p.241-258, 1986.

MORCERF, F.A.P. Ecocardiografia unibidimensional, transesofágica e Doppler. 2.ed. Rio de janeiro: Revinter, 1996. 671p.

MUZZI, R.A.L.; ARAÚJO, R.B.; MUZZI, L.A.L. et al. Ecocardiografia modo $\mathrm{M}$ em cães normais da raça Pastor Alemão (origem americana) do canil da Policia Militar do estado de Minas Gerais. Rev. Ciên. Rural, v.30, p.819-824, 2000.

MUZZI, R.A.L.; ARAÚJO, R.B.; MUZZI, L.A.L. et al. Regurgitant jet area by Doppler color flow mapping: quantitative assessment of mitral regurgitation severity in dogs. J. Vet. Cardiol. V.5, p.33-38, 2003.

NISIMHURA, R.A.; ABEL, M.D.; HATLE, L.K. et al. Assessment of diastolic function of the heart: background and current applications of doppler echocardiography. Part II. Clinical studies. Mayo Clin. Proc., v.64, p.181-203, 1989.

PIPERS, F.S.; HAMLIN, R.L. Clinical use of echocardiography in the domestic cat. J. Am. Vet. Med. Assoc., v.176, p.57-61, 1980.

SANTILLI, R.A.; BUSSADORI, C. Doppler echocardiographic study of left ventricular diastole in non-anaesthetized healthy cats. Vet. J., v.153, p.203-215, 1998 .

SKRODZKI, M.; SCHILLE, S. Doppler echocardiographic examinations in kittens and adult cats. Kleintierpraxis, v.44, p.733-758, 1999.

TRIM, C.M. Sedation and anesthesia. In: HOLZWORTH, J. Diseases of the cat. Philadelphia: W.B. Saunders, 1987. v.1, cap.3, p.43-67.

YU, C.M.; SANDERSON, J.E. Right and left ventricular diastolic function in patients with and without heart failure: effect of age, sex, heart rate, and respiration on Doppler-derived measurements. Am. Heart J., v.134, p.426-434, 1997.

YUILL, C.D.; O'GRADY, M.R. Doppler-derived velocity of blood floww across the cardiac valves in the normal dog. Can. J. Vet. Res. v.55, p.185-192, 1991. 